\title{
Cardiovascular disease, therapy, and mortality of oligosymptomatic and symptomatic patients infected with SARS-CoV-2: experiences of a designated hospital in Poland
}

\author{
Agnieszka Pawlak ${ }^{1,2}$, Karol Dreżewski', Piotr Szymański1,3, Błażej Nowak, \\ Artur Zaczyński' , Zbigniew Król'1, Waldemar Wierzba', Robert Gil11,2 \\ 1 Central Clinical Hospital of the Ministry of the Interior and Administration, Warsaw, Poland \\ 2 Mossakowski Medical Research Centre, Polish Academy of Sciences, Warsaw, Poland \\ 3 Centre of Postgraduate Medical Education, Warsaw, Poland
}

Correspondence to: Agnieszka Pawlak, MD, PhD, Central Clinical Hospital of the Ministry of the Interior and Administration, Interventional Cardiology Department, ul. Wołoska 137, 02-507 Warszawa, Poland phone: +48225081100 , email: agnieszka.pawlak@cskmswia.pl Received: June 22, 2020. Revision accepted: September 1, 2020. Published online: September 8, 2020. Kardiol Pol. 2020; 78 (11): 1162-1165 doi:10.33963/KP.15596

Copyright by the Author(s), 2020
Introduction The coronavirus disease 2019 (COVID-19) pandemic caused by a novel severe acute respiratory syndrome coronavirus 2 (SARS-CoV-2) remains a challenging problem for public health worldwide. The dynamics of the COVID-19 outbreak in Poland seems to be distinct from other European countries. ${ }^{1}$ Available data suggest that severe SARS-CoV-2 infection is associated with an increased inflammatory response and coagulopathy manifesting as prothrombotic state, as well as comorbidities. ${ }^{2-4}$ It is postulated that, if not contraindicated, venous thromboembolism prophylaxis should be administered to every patient admitted to the hospital as per standard of care. ${ }^{5}$ Therefore, it seems interesting whether laboratory markers of inflammation and prothrombotic state together with comorbidities occurred at similar rates in Polish patients with COVID-19.

The aim of this study was to evaluate clinical and laboratory parameters in patients with COVID-19 at the time of admission to the hospital and assess them in relation to the treatment and short-term prognosis.

Methods Study design and participants A total of 166 consecutive patients hospitalized between March and May, 2020 at the Central Clinical Hospital of the Ministry of the Interior and Administration in Warsaw (one of the facilities designated to solely treat patients with COVID-19) were enrolled in the study. They fulfilled the following criteria: 1 ) a positive reverse transcriptase-polymerase chain reaction (RT-PCR) test result for SARS-CoV-2, 2) age 18 years or older. Patients were divided into 3 groups: group I including oligosymptomatic patients who underwent 1-day stay in the hospital emergency room; group II including symptomatic patients who have survived the hospitalization; and group III including symptomatic patients who have died during the hospitalization.

Data collection Available data were collected from the hospital's electronic database using a standard data collection questionnaire. For laboratory parameters, the earliest possible sample was collected, with the day of admission considered the model time point. The National Early Warning Score (NEWS) was calculated for all included patients. ${ }^{6}$ The recovery from COVID-19 was defined as 2 negative RT-PCR test results.

Statistical analysis Statistical analysis was performed with SPSS Statistics for Macintosh, version 13.0 (SPSS Inc., Chicago, Illinois, United States). Group data are expressed as means and (SD), medians and interquartile ranges (IQR), or numbers and percentages, as appropriate. The Shapiro-Wilk test was used to assess the normal assumption distribution. The intergroup differences for categorical variables were 
TABLE 1 Characteristics of patients with coronavirus disease 2019

\begin{tabular}{|c|c|c|c|c|c|}
\hline Parameter & All patients $(n=166)$ & Group I $(n=22)$ & Group II ( $n=75)$ & Group III $(n=69)$ & $P$ value \\
\hline Male sex & $85(51)$ & $8(36)$ & $37(49)$ & $40(53)$ & $0.19^{f}$ \\
\hline Age, y, median (IQR) & $67(54-80)$ & $47(29-57)$ & $60(43-72)^{a}$ & $80(69-86)^{b, c}$ & $<0.001^{g}$ \\
\hline Age $\geq 65$ years & $91(55)$ & $2(9)$ & $27(36)$ & $62(90)^{b, c}$ & $<0.001^{f}$ \\
\hline Hospitalization, d, median (IQR) & $12(4-20)$ & $1(1-1)$ & $19(14-28)^{a}$ & $8(4-14)^{b, c}$ & $<0.001^{g}$ \\
\hline NEWS, median (IQR) & $1(0-3)$ & $0(0-1)$ & $0(0-1)$ & $3(2-4)^{b, c}$ & $<0.001^{9}$ \\
\hline WBC, $1000 / \mathrm{mm}^{3}$, median (IQR) & $6.6(5-9.1)$ & $6.0(4.9-7)$ & $5.9(4.7-7.7)$ & $7.9(5.6-12.2)^{b, c}$ & $<0.001^{\mathrm{g}}$ \\
\hline CRP, mg/l, median (IQR) & $37.2(9.5-112.6)$ & $4.8(1.3-20)$ & $19.2(6.2-54.0)$ & $102.0(46.9-176.4)^{b, c}$ & $<0.001^{9}$ \\
\hline $\begin{array}{l}\text { Procalcitonin, ng/ml, } \\
\text { median (IQR) }\end{array}$ & $2.80(0.10-1.14)$ & $0.1(0.05-0.1)$ & $0.14(0.1-0.46)^{\mathrm{a}}$ & $0.96(0.38-2.5)^{\mathrm{b}, \mathrm{c}}$ & $<0.001^{9}$ \\
\hline D-dimer, $\mu \mathrm{g} / \mathrm{l}$, median (IQR) & $1694(756-2532)$ & $431(340-521)$ & 1062 (514-1985) & $2163(1600-3926)^{c}$ & $<0.001^{9}$ \\
\hline D-dimer $>500 \mu \mathrm{g} / \mathrm{l}$ & $135(81)$ & $11(50)$ & $60(85)$ & $64(95)$ & 0.064 \\
\hline PLT, 1000/mm³, median (IQR) & $201(159-262)$ & $188(154-232)$ & $211(167-264)$ & $195(140-264)$ & $0.58^{g}$ \\
\hline APTT, s, median (IQR); n & $34.4(30.3-38.4) ; 102$ & $\mathrm{n}=0$ & $32.8(29.3-36.6) ; 49$ & $35.4(31.2-40.2) ; 53$ & $0.10 \mathrm{~g}$ \\
\hline PT, s, median (IQR); n & $14.2(12.7-16.6) ; 110$ & $12.9(11.9-14.8) ; 5$ & $13.7(12.3-16.6) ; 47$ & 14.7 (13.2-16.7); 58 & $0.08^{g}$ \\
\hline \multicolumn{6}{|l|}{ Comorbidities } \\
\hline Hypertension & $79(47)$ & $5(23)$ & $30(40)$ & $44(64)^{b, c}$ & $<0.001^{f}$ \\
\hline Diabetes & $32(19)$ & $1(5)$ & $13(17)$ & $18(26)$ & $0.06^{f}$ \\
\hline Coronary artery disease & $50(30)$ & $1(5)$ & $17(23)$ & $32(46)^{b, c}$ & $<0.001^{f}$ \\
\hline \multicolumn{6}{|l|}{ Number of comorbidities } \\
\hline Without disease & $37(22)$ & $16(72)$ & $19(25)^{\mathrm{a}}$ & $2(3)^{b, c}$ & $<0.001^{f}$ \\
\hline 1 disease & $24(14)$ & $3(13)$ & $15(20)$ & $6(9)$ & \\
\hline 2 diseases & $34(20)$ & $1(5)$ & $17(23)$ & $16(23)$ & \\
\hline$\geq 3$ diseases & $71(42)$ & $2(10)$ & $24(32)$ & $45(65)$ & \\
\hline \multicolumn{6}{|l|}{ Treatment } \\
\hline Chloroquine & $118(70)$ & 0 & $58(77)^{\mathrm{a}}$ & $60(87)^{b}$ & $<0.001^{f}$ \\
\hline Lopinavir + ritonavir & $26(15)$ & 0 & $5(7)$ & $21(30)^{\mathrm{b}, \mathrm{c}}$ & $<0.001^{f}$ \\
\hline LMWH & $86(51)$ & 0 & $39(52)^{\mathrm{a}}$ & $47(68)^{b}$ & $<0.001^{f}$ \\
\hline Oxygen therapy & $94(56)$ & 0 & $36(48)^{\mathrm{a}}$ & $58(84)^{b, c}$ & $<0.001^{f}$ \\
\hline Mechanical invasive therapy & $26(15)$ & 0 & $5(7)$ & $21(30)^{b, c}$ & $<0.001^{f}$ \\
\hline Death & $69(42)$ & 0 & 0 & $69(100)^{\mathrm{b}, \mathrm{c}}$ & $<0.001^{f}$ \\
\hline $\begin{array}{l}\text { Time to negative conversion of } \\
\text { SARS-CoV-2 RNA, d, mean (SD) }\end{array}$ & $23(9)$ & $23(7)^{d}$ & $23(11)^{\mathrm{e}}$ & - & $0.96^{\mathrm{h}}$ \\
\hline
\end{tabular}

Data are presented as number (percentage) of patients unless otherwise indicated.

a $P$ value $<0.05$ between group I and II

b $P$ value $<0.05$ between group II and III

c $P$ value $<0.05$ between group I and III

d RT-PCR test for SARS-CoV-2 was performed according to the National Institute of Public Health-National Institute of Hygiene guidelines

e RT-PCR test for SARS-CoV-2 was performed 2 days after clinical stabilization, but not earlier than 7 days from the date of the last positive result

f Fisher exact test

g Kruskal-Wallis test

h $t$ test

Abbreviations: APTT, activated partial thromboplastin time; CRP, C-reactive protein; IQR, interquartile range; LMWH, low-molecular-weight heparin; NEWS, National Early Warning Score; PLT, platelets; PT, prothrombin time; WBC, white blood cells 
assessed with the Fisher exact test, the continuous variables were assessed with the KruskalWallis test or $t$ test. The Fisher test and MannWhitney test with Bonferroni correction were used for multiple comparisons. The time to death in particular subgroups was compared using the Cox-Mental log-rank test. The Cox proportional hazard model was used to assess several risk factors simultaneously. The area under receiver operating characteristic (ROC) curve (AUC) analysis was used to indicate accuracy for mortality prediction for selected variables. The AUC with 95\% CI was calculated for the ROC curve. The Youden index was used to obtain cutoff points for continuous variables. A $P$ value of less than 0.05 was considered significant.

Results and discussion General characteristics of the study population are shown in TABLE 1. Patients in group I, compared with patients in groups II and III, were younger (median [IQR] age 47 (29-57) years; 60 (43-72) years; and 80 (69-86) years, respectively; $P<0.001)$. Only $9 \%$ patients from group I were aged 65 years or older. Conversely, $90 \%$ of patients from group III were in this age range.

The median (IQR) NEWS was lower in groups I and II as compared with group III (0 [0-1]; 0 [0-1]; and 3 (2-4), respectively; $P<0.001)$. Also, these patients had comorbidities less often (hypertension, diabetes, or coronary artery disease [CAD]; 72\%; 25\%; and 3\%, respectively; $P<0.001$ ).

There was no difference in hypertension and CAD prevalence between groups I and II but occurred between group I and groups II and III $(P=0.01$ and $P=0.004$ for hypertension and $\mathrm{CAD}$, respectively).

Laboratory investigations showed lower inflammatory and coagulation parameters in group I than groups II and III, including white blood cell count $(P<0.001)$, $C$-reactive protein (CRP, $P<0.001$ ), procalcitonin (PCT, $P<0.001$ ), and $\mathrm{D}$-dimer levels $(P<0.001)$. The highest values were observed in group III. The area under the ROC curves were 0.88 (95\% CI, 0.780.99), 0.80 (95\% CI, 0.74-0.89), and 0.82 (95\% CI, 0.75-0.91) for age, CRP levels, and PCT levels, respectively. The single cutoff age and concentrations of CRP and PCT were 65 years, $33.5 \mathrm{mg} / 1$, and $1.3 \mathrm{ng} / \mathrm{ml}$, respectively.

In the multivariable Cox regression analysis, only older age (hazard ratio [HR], 1.06; 95\% CI, $1.03-1.09$; $P=0.001$ ), CRP (HR 1.00; 95\% CI 1.00$1.01 ; P=0.001)$ and NEWS of 2 or greater (HR, $1.2 ; 95 \%$ CI, 1.07-1.30; $P=0.002$ ) were found to be risk factors for death.

Group I did not receive any of the analyzed treatments, including patients with comorbidities (CAD, hypertension, diabetes) and elevated D-dimer levels. Lopinavir / ritonavir, oxygen therapy, and mechanical ventilation were used more frequently in group III than in group II (all $P<0.001)$. There was no difference between chloroquine and low-molecular-weight heparin (LMWH) in these groups. A prophylactic dose of low-molecular-weight heparin was used only in groups II and III, in $94 \%$ of patients with increased D-dimer level.

There was no difference regarding the time needed for negative conversion of viral RNA tests in patients hospitalized for 1 day and those hospitalized longer.

The initial analysis showed that our patients, while not discriminated by laboratory tests such as platelet count, activated partial thromboplastin time (APTT), and prothrombin time (PT), differed in regard to 4 parameters: white blood cell count, CRP, PCT, and D-dimer levels, with the latter considered by previous studies to be of paramount importance. ${ }^{7-8}$ Cutoff values for unfavorable prognosis were determined for CRP, senior age, and NEWS. Interestingly, elevated D-dimer levels ( $>500 \mu \mathrm{g} / \mathrm{l})$ were observed in $50 \%$ of patients in group I. It may confirm the increased activity of the coagulation system in patients with COVID-19, as indicated in the literature. The outcome of these patients was good despite no use of anticoagulants. On the other hand, there is a question if the prophylactic dose of LMWH was sufficient for patients in group III, with significantly higher level of D-dimer than in groups I and II. Based on the available literature, as well as our experience, the prophylactic dose of LMWH should be used. ${ }^{9}$

Current literature search revealed that $3 \mathrm{ma}-$ jor comorbidities associated with poor COVID-19 prognosis can be distinguished: hypertension, diabetes, and CAD. ${ }^{8}$ We have likewise observed a higher prevalence of these diseases in our population, with majority observed in patients with severe COVID-19. Despite group I being younger and generally healthier, more than 1 of the assessed diseases was still observed in $15 \%$ of these patients. In this group, hypertension occurred more often than CAD or diabetes. In contrast, $\mathrm{CAD}$ was most prevalent in the group of patients who died during hospitalization (group III).

We have also found it intriguing that both groups I and II were similar with regards to time needed for negative conversion of viral RNA tests; this could be attributed to imperfection of polish Chief Sanitary Inspectorate and Polish Institute of Hygiene in terms of prompt sampling of patients with COVID-19.

Our research indicates that: 1) Oligosymptomatic patients with COVID-19 and comorbidities (cardiovascular disease) but NEWS of 1 or less do not need hospitalization, targeted SARS-CoV-2 therapy, nor thromboprophylaxis. 2) Symptomatic patients with comorbidities, NEWS of 3 or greater, older age, and increased inflammatory markers need hospitalization and targeted therapy. 3) Elevated D-dimer levels are 
present in both oligosymptomatic patients who did not need hospitalization as well as symptomatic patients who were hospitalized.

The major limitation is, since it was a retrospective cohort study in a single center, that there were only 166 patients with COVID-19 included. A multicenter prospective study is needed to further clarify the value of our findings.

\section{ARTICLE INFORMATION}

CONFLICT OF INTEREST None declared

OPEN ACCESS This is an Open Access article distributed under the terms of the Creative Commons Attribution-NonCommercial-NoDerivatives 4.0 International License (CC BY-NC-ND 4.0), allowing third parties to download articles and share them with others, provided the original work is properly cited, not changed in any way, distributed under the same license, and used for noncommercial purposes only. For commercial use, please contact the journal office at kardiologiapolska@ptkardio.pl.

HOW TO CITE PawlakA, Dreziewski K, SzymańskiP, et al. Cardiovascular disease, therapy and mortality of oligosymptomatic and symptomatic patients infected with SARS-CoV-2: experiences of a designated hospital in Poland. Kardiol Pol. 2020; 78: 1162-1165. doi:10.33963/KP.15596

\section{REFERENCES}

1 Raciborski $F$, Pinkas J, Jankowski M, et al. Dynamics of the coronavirus disease 2019 outbreak in Poland: an epidemiological analysis of the first 2 months of the epidemic. Pol Arch Intern Med. 2020; 130: 615-621.

2 Henry BM, Vikse J, Benoit S, et al. Hyperinflammation and derangement of renin-angiotensin-aldosterone system in COVID-19: a novel hypothesis for clinically suspected hypercoagulopathy and microvascular immunothrombosis. Clin Chim Acta. 2020; 507: 167-173.

3 Fogarty H, Townsend L, Ni Cheallaigh C, et al. COVID-19 coagulopathy in Caucasian patients. Br J Haematol. 2020; 189: 1044-1049.

4 Cui $S$, Chen S, Li X, et al. Prevalence of venous thromboembolism in patients with severe novel coronavirus pneumonia. J Thromb Haemost. 2020; 18: 1421-1424.

5 Tang N, Bai H, Chen X, et al. Anticoagulant treatment is associated with decreased mortality in severe coronavirus disease 2019 patients with coagulopathy. J Thromb Haemost. 2020; 18: 1094-1099.

6 Royal College of Physicians. National Early Warning Score (NEWS): standardising the assessment of acute-illness severity in the NHS. RCP, London. 2012. https://www.rcplondon.ac.uk/file/32/download?token=5NwjEyTq. Accessed May $25,2020$.

7 Tang N, Li D, Wang X, et al. Abnormal coagulation parameters are associated with poor prognosis in patients with novel coronavirus pneumonia. J Thromb Haemost. 2020; 18: 844-847.

8 Zhou F, Yu T, Du R, et al. Clinical course and risk factors for mortality of adult inpatients with COVID-19 in Wuhan, China: a retrospective cohort study. Lancet. 2020; 395: 1054-1062.

9 Perica's JM, Hernandez-Meneses M, Sheahan TP, et al. on behalf of the Hospital Clinic Cardiovascular Infections Study Group. COVID-19: from epidemiology to treatment. Eur Heart J. 2020; 41: 2092-2108. 